\title{
An Integro-Differential Model of Language Competition
}

\author{
Mallory Gaspard \\ gaspam3@rpi.edu \\ Peter Craig \\ craigp@rpi.edu \\ Erik Bergland \\ bergle@rpi.edu \\ Department of Mathematical Sciences, Rensselaer Polytechnic Institute
}

April 2, 2019

\begin{abstract}
We study the language shift and competition between the twelve most prominent world-languages while accounting for factors affecting these trends such as governmental influences, migration between nations, and the interaction between competing languages. To model these effects, we propose an integro-differential equation, which is a partial differential equation (PDE), that takes the aforementioned factors into account and predicts the fate of these languages with regards to time and geography. We also carry out a stability analysis of our proposed model under certain circumstances.

In the first part of the investigation, following the establishment of our integro-differential equation model, we also construct a weighted digraph in Python using the United Nations Migrant Data from 19902017 to identify the geographic locations and languages that act as keystones in the global language network. In addition, we execute a numerical simulation of our PDE model in Python, to model the projected future language shifts over time and compare the results from our model to the centrality calculations carried out on our digraph. From the numerical simulations, we predict that the number of monolingual Hindustani speakers will show the greatest growth. Also in terms of the number of first language speakers, English will pass Spanish and Russian will pass Bengali. Furthermore, from our model, it is estimated that in the next fifty years, we can expect to see a rise in the number of English speakers, which will remain clear second beneath Mandarin. We can also expect to see a decrease in the number of Bengali speakers.
\end{abstract}




\section{Contents}

1 Introduction 94

1.1 Background . . . . . . . . . . . . . . . . . . . . . . . . . . . 94

1.2 Objectives $\ldots \ldots \ldots \ldots$

2 Models 95

2.1 Abrams-Strogatz Language Competition Model . . . . . . . . 96

2.2 Walters Language Competition Model . . . . . . . . . . . . . 97

2.3 Our Model . . . . . . . . . . . . . . . . . . . . . . . . . . . . . . . . . 98

$\begin{array}{lll}3 & \text { Analysis } & 100\end{array}$

$3.1 \quad$ Stability Analysis . . . . . . . . . . . . . . . . . . 100

\begin{tabular}{|lll}
\hline & Implementation of the Model & 101
\end{tabular}

4.1 Global Language Graph and Migration Digraph . . . . . . . . 101

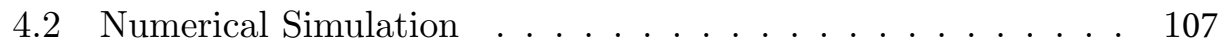

$4.2 .1 \quad$ Methodology . . . . . . . . . . . . . . . . 107

4.2 .2 Assumptions . . . . . . . . . . . . . . . . . . 107

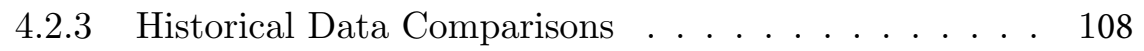

4.3 Simulation Results . . . . . . . . . . . . . . . . . . . . . . 108

4.4 Spatial Dependence. . . . . . . . . . . . . . . . . . . . . . . . 110

4.5 Bilingual Populations . . . . . . . . . . . . . . . . . 111

$4.6 \quad$ Limitations and Future Improvements . . . . . . . . . . . . . 111

$\begin{array}{lll}5 & \text { Conclusions } & 111\end{array}$

\begin{tabular}{lll}
\hline & Acknowledgements & 113
\end{tabular}

\begin{tabular}{lll}
\hline 7 & References & 114
\end{tabular}

8 Appendix A- Proof of Stability 117 


\section{Introduction}

Using provided data on twelve major world languages that includes the number of native speakers and secondary speakers for each, we will develop a model that predicts the changes in native and total language speakers over time, while accounting for government influences, migration, and geography. For further insight into the geographical connections between language speaking groups as well as previously reported migration trends, we also construct a weighted digraph in Python using the Python libraries, NetworkX and the Basemap Toolkit in Matplotlib, to glean information about which geographical areas and countries are key players in determining language trends.

From our model, we are able to determine both the shifts in language speakers over time as well as the geographical locations crucial to determining these changes. Based on our simulations, recognizing that the United States and China have a dominant influence, we have determined that the other countries that contribute significantly to determining language shifts and trends are Brazil, India, France, Spain, Russia, and Saudi Arabia. We also note that France, Spain, and India are particularly advantageous since these nations were found to be key geographical areas to the transitions that take place in the distribution of language speakers. These findings are a result of additional analysis of the structure of the world's migration patterns.

Overall, these results provide rich insight into not only the dynamics of language shift and competition, but also the projected trends in behavior of our globalized world. Taking these results into consideration, greater light is shed on the ways in which we as global citizens can be the most effective and helpful to each other in the long run.

\section{$1.1 \quad$ Background}

Following the conclusion of World War Two, globalization has been increasing, technology has been developing rapidly, and people from all cultures are becoming increasingly interconnected [23]. Today, there are over sixthousand spoken languages on Earth, however, many of Earth's inhabitants primarily speak one of twelve languages, presented in the table below. With the rate and influence of globalization increasing, projecting the trends and shifts in language speakers across the globe becomes essential to the successful operation of the global workforce, as well as the effectiveness of business, 
government, and policy.

\begin{tabular}{|c|c|}
\hline Language & Number of Native (Primary) Speakers (Millions) \\
\hline Mandarin Chinese & 897 \\
Spanish & 436 \\
English & 371 \\
Hindi/Urdu & 329 \\
Arabic & 290 \\
Bengali & 242 \\
Portuguese & 218 \\
Russian & 153 \\
Punjabi & 148 \\
Japanese & 128 \\
French & 76.7 \\
Malay & 23.1 \\
\hline
\end{tabular}

Table 1: Commonly spoken languages on Earth and number of native primary speakers in millions. [31]

\subsection{Objectives}

We aim to develop a comprehensive model that will predict global language shifts and trends over time. Using provided data on twelve major world languages that includes the number of native speakers and secondary speakers for each, we will develop a model that predicts the changes in native and total language speakers over time, while accounting for government influences, migration, and geography. For further insight into the geographical connections between language speaking groups as well as previously reported migration trends, we also construct a weighted digraph in Python using NetworkX and the Basemap Toolkit in Matplotlib, to glean information about which geographical areas and countries are key players in determining language trends.

\section{Models}

In designing our model, we felt it was important to investigate pre-existing language competition models and studies to glean inspiration for our own model as well as identify areas that it can improve upon. In the primary cases we studied, language competition is modeled with differential equations that ultimately describe the manner in which languages under certain 
circumstances either take prominence over another language, or become absorbed completely by a dominant language.

\subsection{Abrams-Strogatz Language Competition Model}

One of the most prominent language competition models is the AbramsStrogatz Model [2]. This model is constructed on the assumption that each language is a fixed entity, and that an individual will not switch from speaking a certain language to speaking a language that has no speakers or is not attractive. This model is given by the ordinary differential equation given below.

$$
\frac{d x}{d t}=y P_{y, x}(x, s)+x P_{x, y}(x, s)
$$

Here, $P_{y, x}=c x^{a} s$ and $P_{x, y}=c(1-x)(1-s)$ are the transition functions governing the shift from speaking one language to another, and $s$ is the status parameter. The status parameter is a real number whose value lies between 0 and 1 and is a measure of the language's attractiveness to potential and current speakers. It encompasses social and economic factors contributing to a language's attractiveness, and its value plays a key role in determining a language's fate.

One of the most significant benefits of the Abrams-Strogatz model is its simplicity and applicability. The Abrams-Strogatz model addresses the probabilistic nature of an individual's decision to switch from speaking one language to another, as well as introducing the status parameter $s$ that takes into account any social and economic factors that affect the speaker's decision to switch from one language to another.

In the relevant studies[27] [20], the parameter $s$ is not easily estimated from real-world data, so it can be difficult to interpret in a modeling context. In many of these studies, $s$ is treated as a single combination of any external factor that affects language spread, including the ones we are considering in our model. Thus, $s$ does not consider the effects of government influence, migration, and language interactions distinctly nor does it consider the potential interaction between the factors.

In addition to the broad nature of the status parameter, the AbramsStrogatz model neglects to account for bilingualism. It inaccurately predicts that bilingual cultures do not exist. The model treats each society as a monolingual group, and neglects to account for societies in which multiple languages are spoken throughout. Furthermore, from the fixed-point analysis, the model has two stable fixed points at $x=0$ and $x=1$. These fixed points predict that two languages in competition can never co-exist, and 
that one will eventually become absorbed by the dominant language. In reality, language shift dynamics are far more complex, as there are more than just two languages in consideration, and it is possible for multiple languages to compete without one completely overtaking the others [2].

\subsection{Walters Language Competition Model}

In 2014, eleven years after the Abrams-Strogatz model became popularized, another language competition model was proposed by Catherine Walters [28], consisting of a system of partial differential equations of reactiondiffusion type. Thus, in order for a one-directional language shift to occur, there must be interaction between the two languages. The Walters model assumes that each language is an independent entity, with one language being dominant over the other. It also assumes that all individuals in the population are monolingual, and like the Abrams-Strogatz model, it defines a language's dominance based upon its status and attractiveness to speakers. Lastly, it assumes that there is no flux at the boundary between geographic regions.

The Walters model is described by equation (2) below.

$$
\left\{\begin{array}{l}
\frac{\partial u}{\partial t}=d_{1} \Delta u+\alpha_{1} u-\beta_{1} u^{2}+\gamma u v \\
\frac{\partial v}{\partial t}=d_{2} \Delta v+\alpha_{2} v-\beta_{2} v^{2}-\gamma u v
\end{array}\right.
$$

In this model, $u(\mathbf{x}, t)$, and $v(\mathbf{x}, t)$ represent the two competing languages, with $u$ being the dominant language, and $\gamma u v$ is a cross term introduced to account for the perceived strength of language $u$ over language $v$. According to the model, $u$ and $v$ will change with space and time, and these languages will diffuse out, with one eventually overtaking the other.

Perhaps the strongest feature of the Walters model is the treatment of each language as an independent entity and the inclusion of the spatial dependence as well as the time dependence of language diffusion. In the Abrams-Strogatz model, spatial dependence was neglected. Thus, the Walters Model is more realistic in terms of the spatial effects on the diffusion and competition of languages.

While this model is useful for predicting the outcome of competition between two languages, one of its weakest features is the a priori assumption that one language is dominant over the other. With that being said, the Walters Model assumes that one language will always benefit from any competition with the other. This assumption may be justified in certain contexts, such as when there is initially a large disparity in speakers. How- 
ever, this assumption precludes the possibility that the formerly submissive language could, in principle, grow to have more speakers than the dominant language. At this point, one would expect that the average speaker would perceive the formerly submissive language as the more attractive option. Since the Walters model does not allow for this possibility, we dispense with that assumption in our model, among other changes.

\subsection{Our Model}

Following the establishment of our assumptions and consideration of the pre-existing language competition models described above, we were able to effectively formulate a single language spread and competition model dependent on space and time, that encompasses distinct factors on language spread such as government influence, migration, and the interaction between languages. In the construction of our model, for mathematical purposes, we identify a few assumptions to note.

- Geographical Assumptions

1. Each geographical region is a rectangle on a grid

2. There is no flux across the boundary of each geographic region, thus, travel between regions is viewed as "teleportation"

- Technological Assumptions

1. Access to internet-based language services such as Google Translate falls within the government influence category

We developed a nonlinear partial differential equation that describes the spread of a language.

$$
\frac{\partial L_{i}}{\partial t}=D_{i} \Delta L_{i}+S\left(x, L_{i}\right), \quad 1 \leq i \leq 12
$$

Where

$$
\begin{gathered}
S(x, \vec{L})=r_{i} L_{i}\left(1-\frac{L_{i}}{K}\right)-G(\vec{x}) \frac{\partial L_{i}}{\partial t}-\epsilon g\left(\frac{\partial L_{i}}{\partial t}\right)+\int_{W} A\left(x-x^{\prime}\right) L_{i}\left(x^{\prime}, t\right) d x^{\prime}+Q(\vec{L}) \\
Q(\vec{L})=\sum_{j \neq i} \gamma_{i, j} \operatorname{sgn}_{\epsilon_{2}}\left(L_{i}-L_{j}\right) L_{i} L_{j}
\end{gathered}
$$

The meaning of each term is displayed in the table below. 


\begin{tabular}{|c|c|}
\hline Term & Significance \\
\hline$L_{i}$ & Language \\
$x$ & Spatial Coordinate \\
$\epsilon$ & Small Parameter (emphasizes that the term it multiplies is small) \\
$K$ & Carrying Capacity of the Earth \\
$G(x)$ & Linear Governmental Influence \\
$g(*)$ & Nonlinear Governmental Influence \\
$A\left(x-x^{\prime}\right)$ & Migration \\
$W$ & The Domain, Represents an approximate world map \\
\hline
\end{tabular}

The main inspiration for the form of equation (1) is the Walters model, as can be seen in the previous section.

When one ignores the nonlinear terms on the right-hand side of that equation, the model reduces to a simple diffusion equation, where the speakers propagate away from their initial locations. Our model also includes this term, and thus this behavior. In addition, the second term on the right hand side deals with how the number of speakers of a given language grows with time. The operational assumption is that the number of speakers increases logistically, which is a standard axiom when analyzing competing populations [1]. Once again, our model retains this term and this behavior. The third and final term in equation (4) indicates what happens when the two languages interact. The term is related to the law of mass action, and states that when both languages are present in an area, speakers convert to the dominant language and leave the dying language at a rate proportional to the product of the number of speakers of both languages. Once again, this interaction term is directly taken from the study of competing populations in ecology, and is the term used to represent predation in the Lotka-Volterra predator-prey equations [4]. As mentioned previously, it is in this term that our model begins to diverge from the one used in the Walters paper. There is a predation-type term for each pair of languages in our model. However, the sign of the interaction is not set a priori, but is determined by the current magnitude of the number of speakers of each language. Thus, if language $i$ has more speakers than language $j$, language $i$ will win over speakers from language $j$.

The terms following the language interaction terms are new additions and have no analogue in the Walters model. The first of these new terms represents the influence that a government can have on the adoption of a language. Often, governments work to maintain the status quo. As such, we model the influence of government as a term which resists large changes in 
the languages spoken within its domain at any given time, i.e. it is a monotonically decreasing, unbounded function of the derivative of the number of speakers of a given language with respect to time. The leading term of this function is linear in the time derivative of the number of speakers, and is hence denoted the "linear government influence." This function does include a spatial dependency, since the governments of the world are not monotone in their resistance to new languages within their borders. For example, the Canadian government actively promotes two languages, English and French.

The final term in equation (4) represents the migration of language speakers. As is explained in more detail, the diffusion of speakers is limited by the presence of no-flux boundary conditions at the borders of each country, as people tend not to leave their home nations simply for the purposes of completing a random walk. Thus, this final term represents purposeful migration from one location to another. The term takes the form of an integral over the entire world map, with the weighting function or propagator $A(x-y)$ representing a probability that an individual will move from location $y$ to location $x$. Thus, the integral calculates the expected number of speakers who will migrate to a given location. As can be seen from its functional form, $A(x-y)$ depends only on the distance between two points in the world, and not any other regional considerations. While there exist many valid criticisms of this assumption, it has been made to simplify the analysis that follows and stands as one of the points which we wish to improve upon with future studies.

\section{Analysis}

\subsection{Stability Analysis}

In order to glean some insight into the potential behaviors of our model, we analyze the stability of a simplified case of our particular integro-differential equations. In this section, we limit the analysis to only two languages, as is common in the literature. Furthermore, we assume that the term representing the government's resistance to change is strictly linear, and does not vary in space. As a final notational convenience, we denote the languages in question as $u$ and $v$ instead of $L_{1}$ and $L_{2}$. Now, it is clear that $u=0, v=0$ are constant steady-state solutions to our model under the assumptions given. Thus, we analyze the global stability of these solutions using the classical energy method, following the procedure given in [28], [22]. Given the assumptions above, our model can be simplified. 


$$
\begin{aligned}
& \frac{\partial u}{\partial t}=D_{1} \Delta u+S_{1}(\mathbf{x}, u, v) \\
& \frac{\partial v}{\partial t}=D_{2} \Delta v+S_{2}(\mathbf{x}, u, v)
\end{aligned}
$$

We define the functions $S_{1}(\mathbf{x}, u, v), S_{2}(\mathbf{x}, u, v)$ as follows.

$$
\begin{aligned}
& S_{1}(\mathbf{x}, u, v)=r_{1} u\left(1-\frac{u}{K}\right)-G \frac{\partial u}{\partial t}+\int_{W} A\left(x-x^{\prime}\right) u\left(x^{\prime}, t\right) d x^{\prime}+\gamma \operatorname{sgn}(u-v) u v \\
& S_{2}(\mathbf{x}, u, v)=r_{2} v\left(1-\frac{v}{K}\right)-G \frac{\partial v}{\partial t}+\int_{W} A\left(x-x^{\prime}\right) v\left(x^{\prime}, t\right) d x^{\prime}-\gamma \operatorname{sgn}(u-v) u v
\end{aligned}
$$

We find that in this reduced model, under certain conditions on the parameters of the problem and the initial data, the solution $u=0, v=0$ can be globally stable. We list the full details of this derivation in Appendix A, as well as the associated conditions mentioned. However, this exercise demonstrates that the model can exhibit stable steady-state behavior, which bodes well for making long-term predictions in the more general case which is used for simulation purposes.

\section{Implementation of the Model}

\subsection{Global Language Graph and Migration Digraph}

While the partial differential equation models language shift and competition over space and time, it is still important to consider the actual connections between geographic regions and countries speaking certain languages. To model the actual connection between speakers of certain languages, we elected to assume a graph-theoretical approach. To begin, we grouped countries by their official language. We considered eleven commonly spoken languages (with respect to primary speakers): Chinese-Mandarin, Spanish, English, French, Portuguese, Hindi, Punjabi, Russian, Arabic, Japanese, and Bengali. In this portion, we did not consider Malay due to it's negligible effects on the network. According to 2018 data from Ethnologue, Malay has far fewer native speakers than the other languages considered in our list and is only concentrated as an official language within four countries around the Malaysian Peninsula [12]. Furthermore, according to recent CIA data from The World Factbook, Malaysia has a low migration rate, which indicates that while a small portion of Malay-speakers are moving away, these effects are negligible compared to the other countries in our study [3]. 
Malay-speaking migrants are primarily leaving Malay-speaking countries for places like Australia and Singapore which are primarily English-speaking and Chinese-speaking countries [25]. Thus, with Malay's highly localized concentration in Southern Asia,small number of native speakers in comparison to others considered in our study, and small movement into Englishspeaking and Chinese-speaking countries, Malay's effects on the network were negligible.

In this portion, for each of the eleven languages, we identified the nations whose primary official language matched the language in consideration [31. Then, we obtained longitude and latitude coordinates for the center of each nation [17]. In Python, we wrote a program employing NetworkX functions and the Basemap Toolkit in Matplotlib to place graph nodes on a world map corresponding to the coordinates of each country's longitudinal and latitudinal centers, and we assigned a specific color to each node according to the official language spoken in that country. Then, to generate the edges, we connected all of the nodes of the same color to each other. Thus, we are left with eleven disjoint, undirected graphs, exhibiting the geographic connections between same-language groups across the globe.

For further insight into the current geographic distribution and the keystone locations of each language, as well as the global migratory trends for the past few decades, we employ the United Nations migrant data for totals between 1990 and 2017. With this, we aim to identify the countries that are essential to the structure of this network and the countries most influential in the language network. By identifying these locations, we will be able to understand the geographical areas historically most responsible for a language's vitality and compare them with results from the numerical simulations to determine which of these locations are most significant in determining shifts in language speaker over time. To begin, we maintained the structure of the graph developed in the previous section, and removed all of its edges. We then parsed the United Nations migration data, and assigned an edge between two countries where inhabitants are involved in migration. For computational purposes, we assume that the migration between countries with fewer than 10,000 migrants per year is negligible. We also assume that the individuals speak the origin country's language, but will switch languages once they become a part of the destination country's population. We assigned a weight on the edge directed from the origin country to the destination country, given in equation (7).

$$
W=\frac{P_{\text {total }}}{10,000}
$$




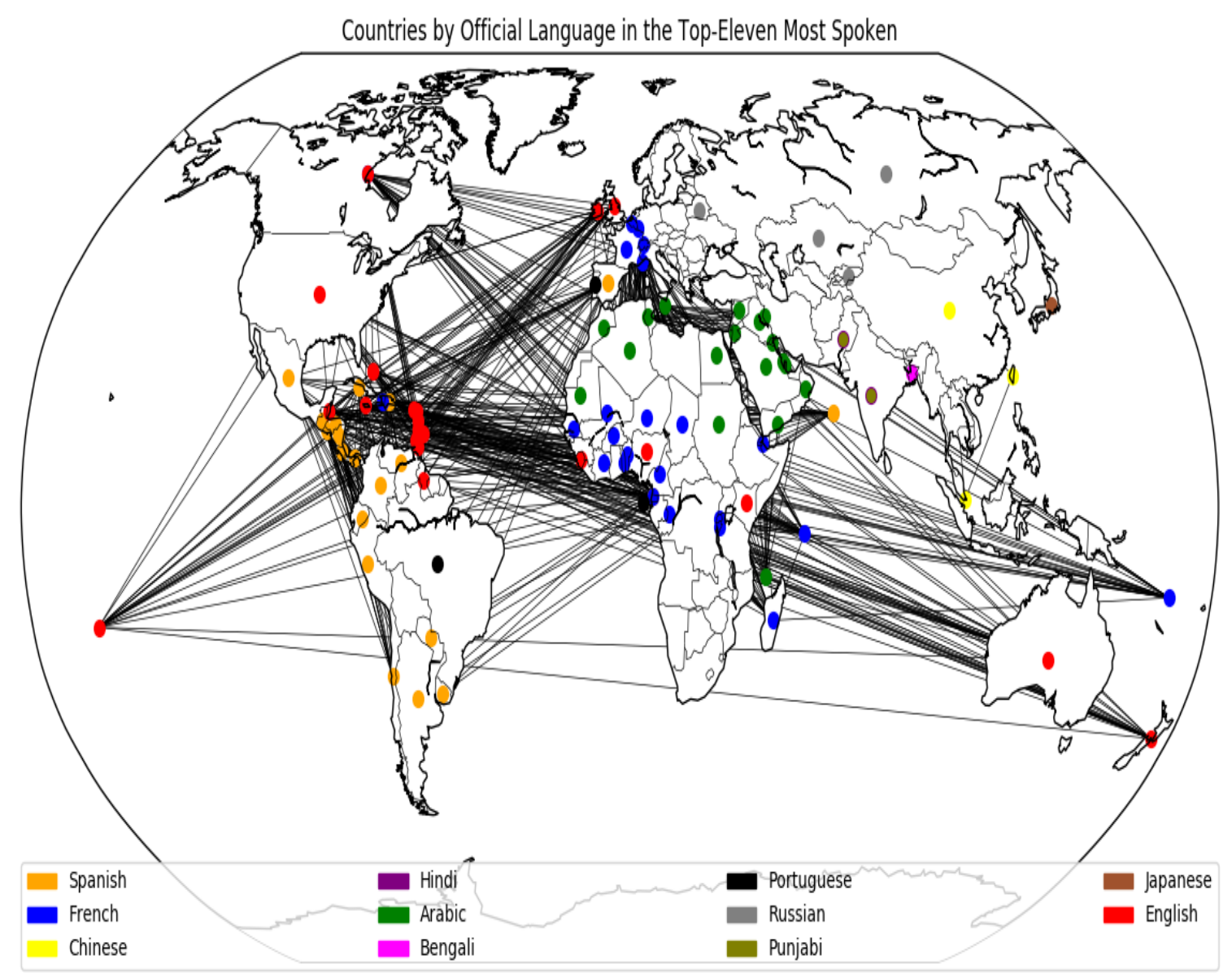

Figure 1: World Map graph with connections between countries with the same official primary language 
Here $P_{\text {total }}$ is the total number of migrants from the origin country to the destination country recorded from 1990 to 2017. Now, with regards to direction, in most cases, migration was strongly one-directional. The number of individuals migrating in the reverse direction is virtually negligible. Therefore, we assigned the edge direction according to the country receiving the higher number of migrants. Thus, weighted edges go into countries receiving the immigrants [26] [17] 21] 35] 30] [13] 14] [11] 33] 32] 34] 10] 86.

Following the generation of the language migration digraph, we are now ready to gain insight into the geographical areas that are essential to language vitality, and which of these countries are currently receiving the most new inhabitants. We investigate two types of centrality, Betweenness Centrality and Eigenvector Centrality, both of which will give us insight into the nodes that are crucial to the structure of this network. The Betweenness Centrality value for each node will help us to identify which locations either hinder or encourage language spread from one language to another based primarily on the amount of individuals choosing to migrate there and the countries they originate from [36]. The Betweenness Centrality value is defined below in equation (8).

$$
c_{b}=\sum_{s, t \in V} \frac{\sigma(s, t \mid v)}{\sigma(s, t)}
$$

Here $s, t$ are nodes in the node set $V, \sigma(s, t)$ is the number of shortest paths between $s$ and $t$ and $\sigma(s, t \mid V)$ is the number of paths passing through a node $v \neq s, t$ [19]. Similarly, the Eigenvector Centrality of the network will help us to identify those countries that are most influential in our migration and language network. The strongest countries will not only receive a significant number of migrants from a greater number of countries, but it will receive migrants from other relatively influential countries as well. In other words, migrants immigrating to a certain country will take their country's influence with them. For example, if migrants choose to leave a country whose immigration numbers are high, they will bring that influence with them to the country they elect to immigrate to. Now, according to the Perron-Frobenius Theorem, the adjacency matrix will have a single largest eigenvalue. In this case, the largest eigenvalue from the graph's adjacency matrix will correspond to the eigenvector used to calculate the network's Eigenvector Centrality [24]. Thus, the Eigenvector Centrality for a given country in the migration-language network is given by equation (9).

$$
\lambda e_{i}=\sum_{j} A_{i j} e_{j}
$$



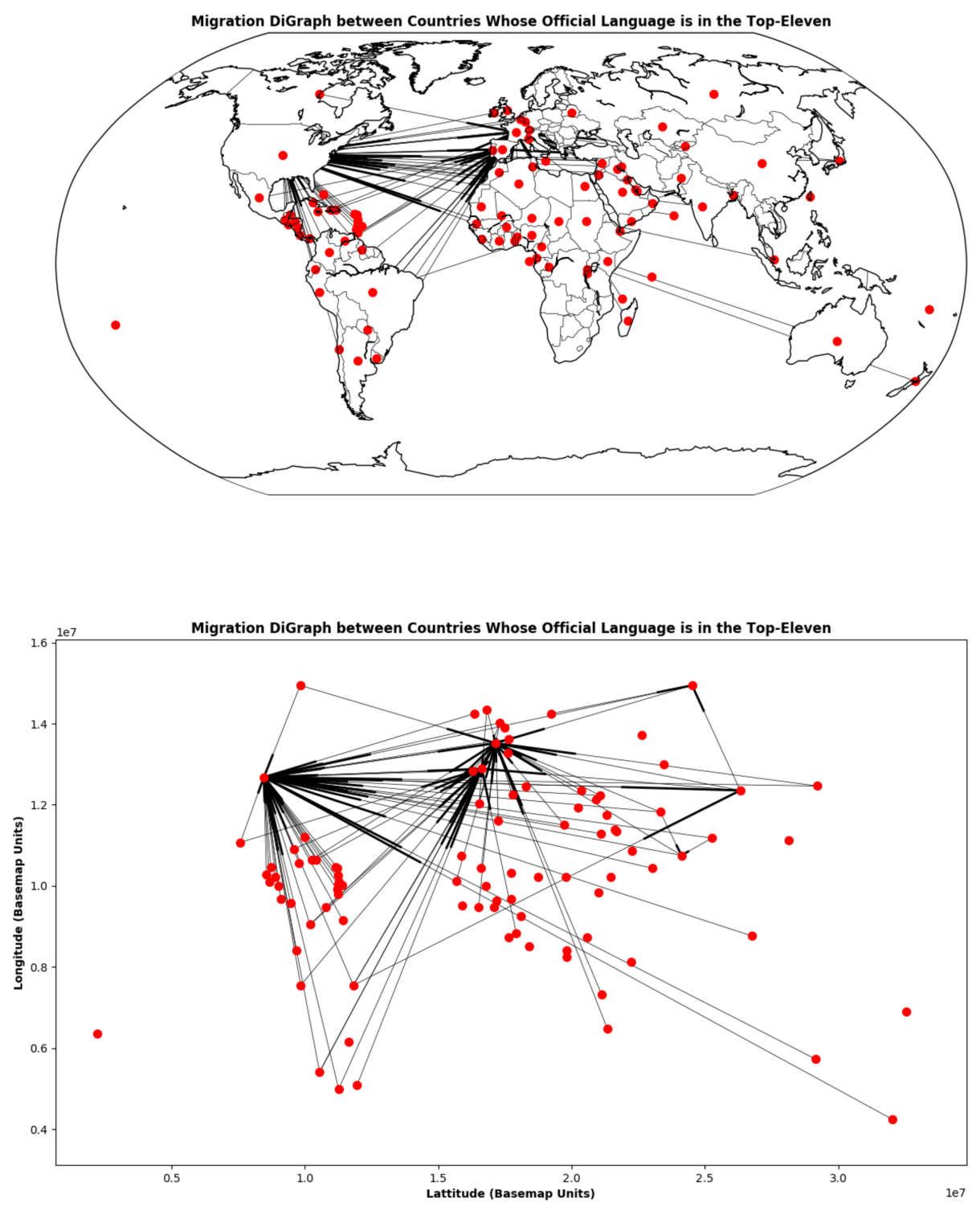

Figure 2: Migration digraph showing collective shifts in population and language concentration from 1990-2017. Note: Both graphs in this image are the same and are on the same scale. 
Here $A_{i j}$ is the adjacency matrix entry corresponding to the connection between the $i^{t h}$ and $j^{t h}$ nodes, and $e_{j}$ is the $j^{\text {th }}$ node's centrality, which is the sum of all of it's connections to the other nodes in the network [5]. In Python, a script was written using the NetworkX module to calculate both the Betweenness Centrality value and Eigenvalue Centrality of each country in the network. In the table below, we present the five countries considered in our digraph with the highest Betweenness Centrality values.

\begin{tabular}{|c|c|c|}
\hline Keystone Nations & Official Language(s) & $c_{b}$ \\
\hline United States & English & 0.0184 \\
China & Chinese (Mandarin) & 0.00638 \\
France & French & 0.00425 \\
Spain & Spanish & 0.00179 \\
India & Hindi and Punjabi & 0.00005599 \\
\hline
\end{tabular}

Table 2: Top-five nations crucial to structure of the language network and language competition based on Betweenness Centrality calculations. These values point out the nations that are essentially "migration hubs," as they have the strongest influence language flow throughout the network.

Similarly, for the Eigenvalue Centrality Results, we present the five countries considered in our digraph with the highest Eigenvalue Centrality values.

\begin{tabular}{|c|c|c|}
\hline Keystone Nations & Official Language(s) & $e_{i}$ \\
\hline France & French & 0.697 \\
United States & English & 0.638 \\
Spain & Spanish & 0.322 \\
China & Chinese (Mandarin) & 0.0476 \\
Russia & Russian & 0.0118 \\
\hline
\end{tabular}

Table 3: Top-five nations most influential in the language network based on Eigenvalue Centrality values. The Eigenvalue Centrality identifies these countries as being the most influential in language shift and migration based upon their immigrants' origin countries, as well as the number of distinct countries that these five countries receive immigrants from.

Now that we have gained appropriate insight into which countries have played the most significant role in language trends over the past twentyseven years, we will be able to couple this information with the results from the numerical simulations of our model and incorporate all of these results into making decisions for our client. 


\subsection{Numerical Simulation}

\subsubsection{Methodology}

Our simulations are heavily based on the language competition integrodifferential equation that we have developed. This equation is slightly modified for the ease of computation and the specifics of these modifications are discussed in the proceeding paragraph., Following the modifications, the simulation was then implemented in countries around the world. The simulations run over a selection of 43 different countries and include 12 of the most heavily used languages in the world.

The primary modification to equation (4) imposed within the numerical simulations is an adjustment to our migration term. Instead of evaluating an integral at every time step, the simulation uses current data to estimate the number of migrants in each country based upon the country's current population. New people arriving in a given country are assumed to have a language distribution identical to the rest of the world, and people leaving a given country are assumed to have the same language distribution as the country they are leaving. In addition, the nonlinear government interaction term in equation (4) is neglected. This is equivalent to setting $\epsilon$ equal to zero.

The resulting equation is solved using a finite element method, in which each country is assigned a grid size based upon its geographical size. Since the solutions in each country are effectively independent, during each time step the status of each country is updated individually, and then new global totals for the number of speakers of each language are calculated.

A government parameter is also present in our simulations. This is estimated when possible using historical data, and otherwise based upon the number of official languages in that county. In particular, countries with a greater number of official languages is assigned a higher government resistance value, which helps the less common languages to survive. Ultimately, this is usually not enough to counteract the tendency for people to switch to a nation's primary language unless there is also considerable support from immigrants to maintain the speaking population. Over time the minor languages tend to only be spoken by bilingual individuals.

\subsubsection{Assumptions}

- All of the language interaction values are the same in order to make the implementation of the numerical simulation computationally simpler. 
- Migration is approximated within the simulation as an appropriate number of people added to the center of a country, which then diffuse throughout. The number of people added is determined by the current rate of migration per thousand people for that country, which we assume to be a constant throughout the simulation.

- between distinct language speaking populations primarily occurs during migration.

- While people who speak more than two languages can be included in the simulations as an additional language-speaking group, they were neglected due to their relative scarcity.

\subsubsection{Historical Data Comparisons}

A good check that our simulations are producing reasonable results can be obtained using historical data on the loss of low-usage languages. Our sample cases include Welsh and Gaelic, both of which have been overrun by English in the last hundred years. There are now no monolingual speakers of either language, which is exactly what our model predicts. The simulations are able to nicely reproduce the historical data for the lost languages, but are a rough approximation to the English data because of various fluctuations in the total population, due to factors like migration [37].

This model is capable of matching the data at least as well as models created in the past, but will be able to better handle predictions of government and social interactions in the rate of degradation for less common languages.

\subsection{Simulation Results}

Our simulations have been run under a variety of different conditions regarding government behavior and interactions between languages. Ultimately the primary impact of variations in these parameters is to determine how quickly less common languages will disappear. The initial conditions have been determined based on any information available about the number of speakers for different languages around the world[34] [29] [15] [16] [30] [32] [10] [13] [14] [8] [9] [7].

All of our simulations indicate that Mandarin will remain the most common native language in the world, primarily because of the large carrying 


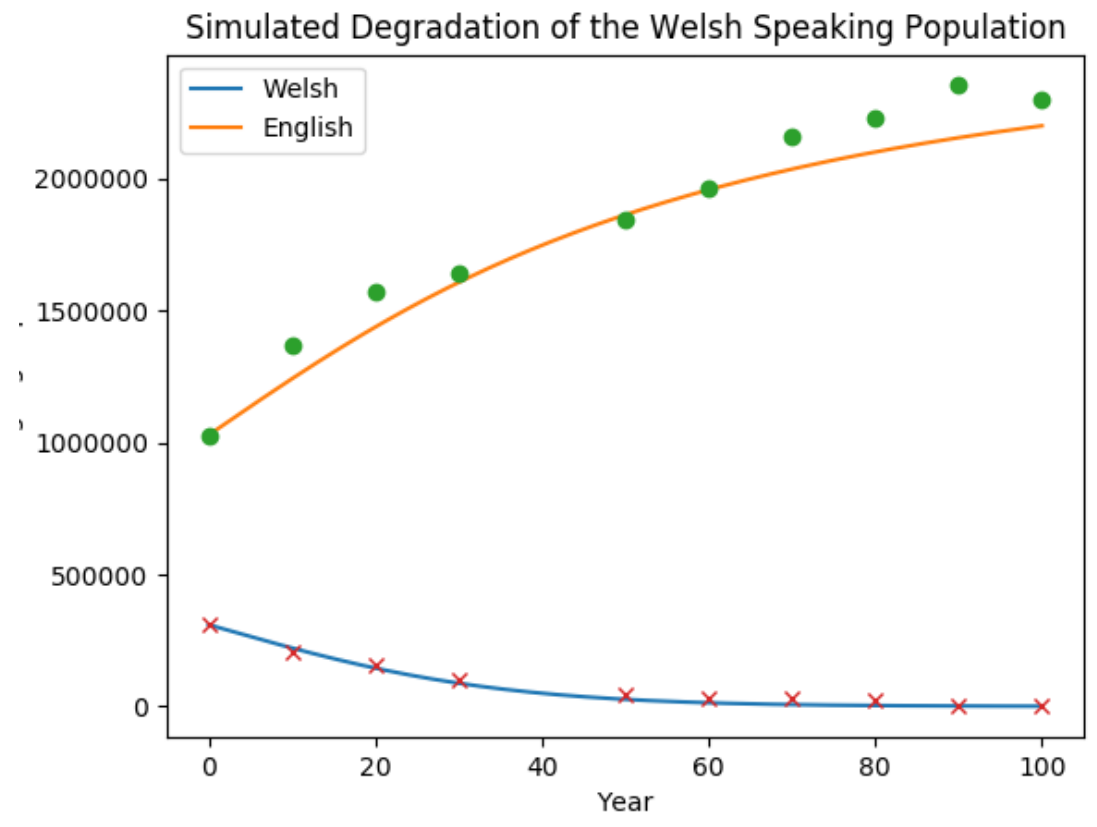

Figure 3: This plot shows the number of primary languages speakers for English and Welsh over a 100 year period. The points indicate historical data, and the two curves are produced by our simulations 
capacity of China, which allows for a large total population. Our simulations also indicate that Bengali will become less significant over time due to the eventual loss of many speakers in India. Since most of that population is predicted to eventually switch to Hindustani, that language eventually surpasses several others. While government support helps to reduce the rate of this degradation, it is not enough to prevent substantial losses. The Spanish and English native speaker populations are also likely to trade places in the future.

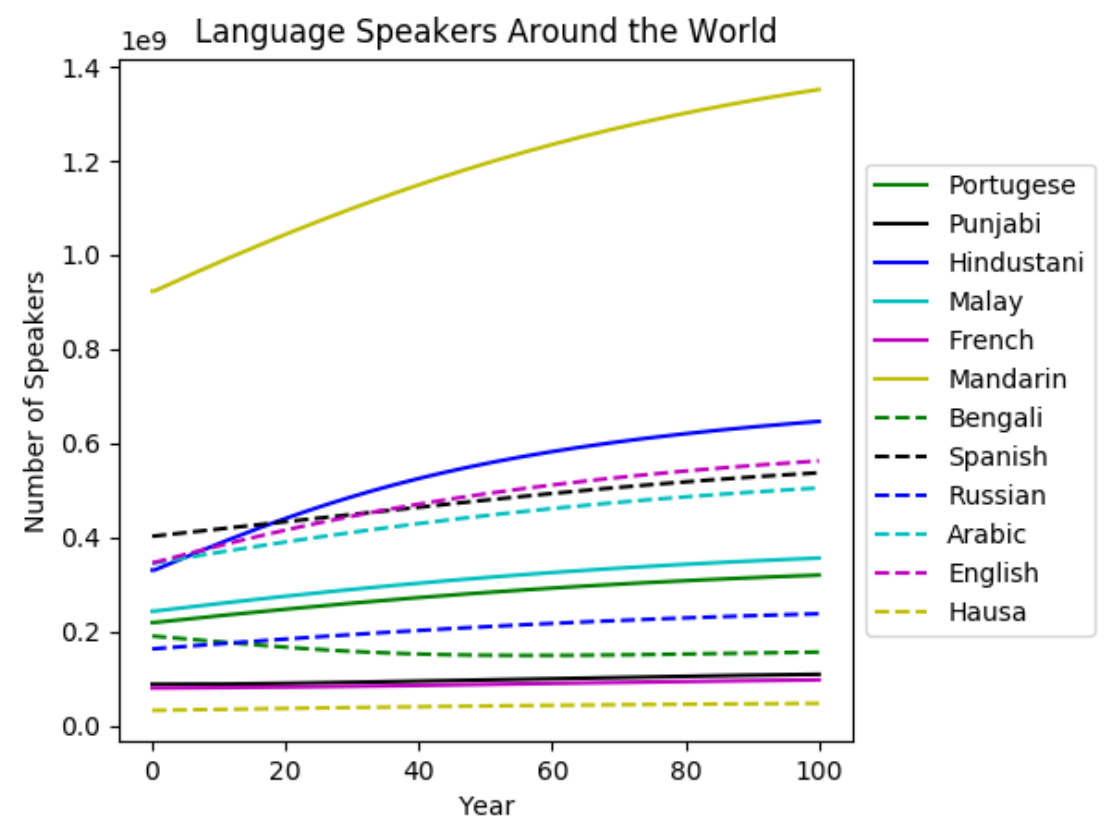

Figure 4: Number of native language speakers for 12 languages over a 100 year interval, which begins at the present day

\subsection{Spatial Dependence}

Our countries are initialized as a rectangular grid, the size of which is determined by the total surface area of the nation. In its current form the spatial dependence of the simulation is not of particular interest. The end effect is that if the migration term is zero, then the distribution of people is uniform throughout the country. If the migration term is not zero, then there is an abundance or a lack of people in the central regions, so the population 
then diffuses as one would expect. Since this behavior is of relatively limited interest for the purposes of this project, we will not include any further analysis of the spatially-dependent behavior.

\subsection{Bilingual Populations}

Our simulations can account for bilingual populations as well as the monolingual populations. These populations behave a bit differently based upon historical data, with less common languages degrading at a much slower rate for bilingual speakers. With this in mind, bilingual populations have a considerably smaller language interaction term, which prevents rapid decay of their population. Especially with migration our simulations indicate that it is quite possible for a bilingual population to remain stable and maintain an uncommon language, at least for periods of a few hundred years. This calming of parameters helps to maintain a higher number of speakers for several languages, and has a large effect on the number of English speakers. Since there are many people who learn English as a second language, including this population makes the language much stronger, and it remains dominant over everything except for Mandarin throughout the simulation.

\subsection{Limitations and Future Improvements}

The biggest limitation of our current model is the migration term, which is designed to manage interactions between different countries. The current model has been adopted primarily for the simplicity of implementation. A reasonable future continuation of this model would be to simulate a more complex migration term, which would really link countries together and create more spatial dependence.

\section{Conclusions}

Our simulations indicate that in terms of native speakers, Bengali will trade places with Russian in terms of relative propensity. This is due to the large portion of the Bengali native speakers who now live in India, where the model predicts a decrease in this population. Russian however tends to be the dominant language in most countries that make a large contribution to the overall number of native speakers, so no substantial portion of this population will decay. By similar reasoning, the simulations predict that the 


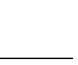

population of natural language English speakers will pass the Spanish population. The largest predicted shift for monolingual speakers is the rise of Hindustani, which becomes the second most common language. Most of this population is present in India, and results from Bengali speakers switching to Hindustani. Including bilingual individuals makes the total Spanish population somewhat more stable, and creates a large boost for English, which then becomes much closer to matching Mandarin, and takes clear second in terms of total speakers. Most other behavior remains the same with the addition of bilingual people.

The simulations have indicated that regions with high population densities and regions receiving a considerable number of immigrants are geographic keystones to global language trends. From the numerical simulations and the graphs, the United States and China are the most influential countries in the network. While these two countries are highly significant, there are several other nations that have proved to be of great importance as well. Based on Betweenness Centrality value, India is a crucial country in the structure of the network, as it has a high population density and receives a large number of migrants. On the other hand, while Brazil is not as crucial to the overall structure and flow within the network based on Betweenness Centrality values, it has a large population and it contributes relatively significantly to migration throughout the language network, as it receives immigrants from and contributes immigrants to highly influential countries such as Spain, China, France, and the United States. In addition, Spain, France, and Russia prove to be crucial countries in the network as is reflected in both the results of the numerical simulation and the digraph.

From the numerical simulation we see that the these regions have a reasonable population density and the vast majority of the population speak a single language. This makes it easy for our representatives to communicate with most of the people in the region. From the digraph, Spain and France have some of the highest Betweenness Centrality and Eigenvector Centrality values, suggesting that they are not only crucial to the network's structure and the flow of language shift, but they are also two of the most influential nations in the network. Thus, they are receiving a high number of immigrants from many distinct countries, and are also receiving immigrants from other influential countries, boosting their overall influence in the migration-language flow. Similarly, Russia has a comparably high Eigenvector Centrality value, validating them as an important player in the network. While Russia does not receive as many immigrants as the other countries in 
the network, they receive a considerable number of immigrants from moreinfluential nations. Geographically, they have European, Middle-Eastern, and Asian influence, making them a fairly central and important location to consider. Finally, although Saudi Arabia's migration totals and centrality values do not suggest that it is as influential as other countries considered in the study, Saudi Arabia receives a fairly significant number of immigrants from a variety of Middle Eastern, Arabic-speaking nations. Its geographic location in the network and its migration totals render it important in considering the overall behavior of the flow throughout the network. Future research in the simulated model will primarily be focused on the additional inclusion of migration effects, with a more realistic model that includes differing migration rates from different regions of the world. This would have an effect on the language distribution of the incoming individuals.

All in all, we have established a practical integro-differential equation model for language shift and competition which accounts for governmental influence, interaction between multiple languages, and migration that is capable of predicting language trends over time. We have also successfully developed a detailed digraph to model the connection between various language speaking populations as well as serve as a reasonable point of comparison with our numerical simulations, to provide an additional resource for predicting language trends. Overall, we have successfully gleaned insight into the intricate patterns and influences governing the fate of the spoken word across the globe.

\section{Acknowledgements}

This work was supported, in part, through the NSF Research Training Groups program (DMS-1344962) and was originally completed as part of the 2018 COMAP Mathematical Contest in Modeling. As such, we would like to extend our special thanks to Professor Peter Kramer of the RPI Mathematics Department for serving as our coach for the contest, as well as the entire Department of Mathematical Sciences at RPI for their continued support. 


\section{$7 \quad$ References}

\section{References}

[1] The logistic equation. Northwestern University, https://sites. math.northwestern.edu/ mlerma/courses/math214-2-03f/notes/ c2-logist.pdf. Last accessed March 16, 2019.

[2] D. M. Abrams and S. H. Strogatz, Modeling the dynamics of language death, Nature, 424 (2003), p. 900. Department of Theoretical and Applied Mechanics, Cornell University, Ithaca, New York 14853, USA.

[3] U. S. C. I. AGency, Malaysia. https://www.cia.gov/library/ publications/the-world-factbook/geos/my.html. Last accessed March 16, 2019.

[4] M.-C. Anisiu, Lotka, volterra and their model, Didactica Mathematica, 32 (2014), pp. 9-17.

[5] P. Bonacich, Power and centrality: a family of measures, American Journal of Sociology, 92 (1987), pp. 1170-1182. University of California, Los Angeles.

[6] A. Bressan, Lecture notes on sobolev spaces. Lecture Notes, February 2012. Penn State University https://www.math.psu.edu/bressan/ PSPDF/sobolev-notes.pdf. Last accessed March 16, 2019.

[7] P. M. O. B. Darussalam, National statistics. Web, November 2016. "https://web.archive.org/web/20161111141102/http: //www.depd.gov.bn/SitePages/National\%20Statistics.aspx. Last accessed March 16, 2019.

[8] G. F. S. David M. Eberhard and C. D. Fennig, Ethnologue bengali. Web. https://www.ethnologue.com/language/ben. Last accessed March 16, 2019.

[9] —, Ethnologue - hausa. Web. https://www.ethnologue.com/ language/hau. Last accessed March 16, 2019.

[10] — Ethnologue - hindi. https://www.ethnologue.com/language/ hin. Last accessed March 16, 2019.

[11] —, Ethnologue - japanese. https://www.ethnologue.com/ language/jpn. Last accessed March 16, 2019. 
[12] — Ethnologue - malay, standard. Web. https://www.ethnologue. com/language/zlm. Last accessed March 16, 2019.

[13] —, Ethnologue - punjabi. https://www.ethnologue.com/ language/pan. Last accessed March 16, 2019.

[14] —, Ethnologue - russian. https://www.ethnologue.com/ language/rus. Last accessed March 16, 2019.

[15] — Ethnologue - spanish. https://www.ethnologue.com/ language/spa. Last accessed March 16, 2019.

[16] O. I. DE LA Francophonie, 2014: La langue francais dans le monde, (2014). https://www.francophonie.org/Langue-Francaise-2014/ projet/Rapport-OIF-2014.pdf. Last accessed March 16, 2019.

[17] G. Developers, Countries. Online open dataset, January 2012. https://developers.google.com/public-data/docs/canonical/ countries_csv. Last accessed March 16, 2019.

[18] C. Fox, An Introduction to the Calculus of Variations, Dover Publications, 1987.

[19] A. A. Hagberg, D. A. Schult, and P. J. Swart, Exploring network structure, dynamics, and function using NetworkX, (2008), pp. 11-15. Pasadena, CA USA.

[20] Y. LiU, Modeling language competition. Online essay, December 2009. University of Illinois - Urbana Champaign, Department of Physics, http://guava.physics.uiuc.edu/ nigel/courses/569/ Essays_Fall2009/files/liu.pdf. Last accessed March 16, 2019.

[21] D. Lyons, How many people speak spanish, and where is it spoken?, Babbel Magazine, (2017).

[22] G. Mulone, B. Straughan, and W. Wang, Stability of epidemic models with evolution, Studies in Applied Mathematics, 118 (2007), pp. 117-132.

[23] U. Nations, Development and globalization: Facts and figures: 2012, Palais des Nations, 8-14, Av. de la Paix, 1211 Geneva 10 Switzerland, April 2012, United Nations Conference on Trade and Development, United Nations. https://unctad.org/en/PublicationsLibrary/ webgdsdsi2012d2_en.pdf. Last accessed March 16, 2019. 
[24] M. Newman, The mathematics of networks, in The New Palgrave Dictionary of Economics, S. Durlauf and L. Blume, eds., vol. 2, Palgrave Macmillan, 2008. Center for the Study of Complex Systems, University of Michigan.

[25] C. R. S. of Department of Immigration and CitizenSHIP, Community information summary: Malaysia-born. Web. https://www.dss.gov.au/sites/default/files/documents/02_ 2014/malaysia.pdf. Last accessed March 16, 2019.

$[26]$ U. N. D. of Economic And S. AfFairs, United nations migrant stock by origin and destination, (2017). New York, New York, USA http://www.un.org/en/development/desa/population/ migration/data/estimates2/estimates17. asp. Last accessed March $16,2019$.

[27] D. Stauffer, X. Costello, V. Eguiluz, and M. S. Miguel, Microscopic abrams-strogatz model of language competition, Physica A: Statistical Mechanics and its Applications, 374 (2007), pp. 835-842.

[28] C. E. WAlters, A reaction-diffusion model for competing languages, Meccanica, 49 (2014), pp. 2189-2206. Department of Mathematical Sciences, Durham University, Durham DH1 3LE, UK.

[29] WiKIPEDIA, List of countries by english-speaking population. https://en.wikipedia.org/wiki/List_of_countries_by_ English-speaking_population. Last accessed March 16, 2019.

[30] — List of countries where arabic is an official language. Data retrieved from respective country's Ethnologue page https://en.wikipedia.org/wiki/List_of_countries_where_ Arabic_is_an_official_language. Last accessed March 16, 2019.

[31] — List of languages by total number of speakers. https: //en.wikipedia.org/wiki/List_of_languages_by_total_number_ of_speakers. Last accessed March 16, 2019.

[32] —, List of territorial entities where china is an official language. https://en.wikipedia.org/wiki/List_of_territorial_ entities_where_Chinese_is_an_official_language. Last accessed March 16, 2019. 
[33] — List of territorial entities where english is an official language. https://en.wikipedia.org/wiki/List_of_territorial_ entities_where_English_is_an_official_language. Last accessed March 16, 2019.

[34] —, List of territorial entities where portugese is an official language. https://en.wikipedia.org/wiki/List_of_territorial_ entities_where_Portuguese_is_an_official_language. Last accessed March 16, 2019.

[35] E. M. Wood, How many people speak french, and where is it spoken?, Babbel Magazine, (2017).

[36] E. YAN, Applying centrality measures to impact analysis: A coauthorship network analysis, Journal of the Association for Information Science and Technology, 60 (2009), pp. 2107-2118. Indiana University.

[37] M. Zhang And T. Gong, Principles of parametric estimation in modeling language competition, Proceedings of the National Academy of Sciences of the United States of America, 110 (2013), pp. 9698-9703. Institute of Linguistics, Shanghai Normal University.

\section{Appendix A- Proof of Stability}

As discussed in the section on stability analysis, the equations for our model can be reduced.

$$
\begin{aligned}
& \frac{\partial u}{\partial t}=D_{1} \Delta u+r_{1} u\left(1-\frac{u}{K}\right)-G \frac{\partial u}{\partial t}+\int_{W} A\left(x-x^{\prime}\right) u\left(x^{\prime}, t\right) d x^{\prime}+\gamma \operatorname{sgn}(u-v) u v \\
& \frac{\partial v}{\partial t}=D_{2} \Delta v+r_{2} v\left(1-\frac{v}{K}\right)-G \frac{\partial v}{\partial t}+\int_{W} A\left(x-x^{\prime}\right) v\left(x^{\prime}, t\right) d x^{\prime}-\gamma \operatorname{sgn}(u-v) u v
\end{aligned}
$$

Since the government influence term has been assumed to be constant, we rescale the time variable so that the influence of the government term is felt in the changed timescale. Introducing the new variable $\tau=(1+G) t$, the government influence term on the right-hand side combines with the time derivative on the left-hand side, yielding equations (12) and (13).

$$
\frac{\partial u}{\partial \tau}=D_{1} \Delta u+r_{1} u\left(1-\frac{u}{K}\right)+\int_{W} A\left(x-x^{\prime}\right) u\left(x^{\prime}, t\right) d x^{\prime}+\gamma \operatorname{sgn}(u-v) u v
$$




$$
\frac{\partial v}{\partial \tau}=D_{2} \Delta v+r_{2} v\left(1-\frac{v}{K}\right)+\int_{W} A\left(x-x^{\prime}\right) v\left(x^{\prime}, t\right) d x^{\prime}-\gamma \operatorname{sgn}(u-v) u v
$$

For the purposes of the analysis, we introduce the following inner product, and its associated norm defined in equations (14) and (15).

$$
\begin{gathered}
(u, v)=\int_{W} u(\mathbf{x}) v(\mathbf{x}) d \mathbf{x} \\
\|u\|=(u, u)^{1 / 2}
\end{gathered}
$$

Additionally, we introduce the following important inequalities which will be used repeatedly in the analysis to follow. They are the CauchySchwartz inequality and the Sobolev inequality.

$$
\begin{gathered}
\int_{W} u(\mathbf{x}) v(\mathbf{x}) d \mathbf{x} \leq\left(\int_{W}(u(\mathbf{x}))^{2} d \mathbf{x}\right)^{1 / 2}\left(\int_{W}(v(\mathbf{x}))^{2} d \mathbf{x}\right)^{1 / 2} \\
\left(\int_{W}(u(\mathbf{x}))^{4} d \mathbf{x}\right)^{1 / 2} \leq c \int_{W}|\nabla u|^{2} d \mathbf{x}
\end{gathered}
$$

Here the constant $c=c(W)$ is a function of the domain $\mathrm{W}$ only. Crucially, the latter inequality holds only for functions that are a member of the Sobolev-Hilbert space $H_{0}^{2}(W)=\{u(\mathbf{x}) \mid u(\mathbf{x})=\nabla u(\mathbf{x})=0, x \in \partial W\}$, so we make the assumption that our solutions inhabit this space at the beginning of the analysis. This space is characterized by the property that the function and its first derivative vanish on the boundary of the domain $\mathrm{W}$ [6]. This fact will be useful in the derivation to follow.

In order to show that the solution tends towards $(u, v)=(0,0)$, we construct a function $E(u, v)$, called an energy function, which is non-negative, monotonically decreasing along the flow of the model, and achieves its minimum at the point $(0,0)$. If we can accomplish this, we will have shown that the flow causes any point to move in a direction that causes the energy function to achieve its minimum, which occurs precisely at the origin. Thus, any disturbances from the origin will ultimately decay. To this end, we define the energy function given in equation (18).

$$
E(u, v)=\frac{1}{2}\left(\|u\|^{2}+\|v\|^{2}\right)
$$


We multiply equation (7) by $u$ and equation (8) by $v$ and integrate over the domain $\mathrm{W}$.

$$
\begin{gathered}
\frac{1}{2} \frac{\partial\|u\|^{2}}{\partial \tau}=D_{1} \int_{W} u \Delta u d \mathbf{x}+r_{1} \int_{W} u^{2} d \mathbf{x}-\int_{W} \frac{u^{3}}{K} d \mathbf{x}+\int_{W} u \int_{W} A\left(x-x^{\prime}\right) u\left(x^{\prime}, t\right) d \mathbf{x}^{\prime} d \mathbf{x} \\
+\int_{W} \gamma \operatorname{sgn}(u-v) u^{2} v d \mathbf{x}
\end{gathered}
$$

Using integration by parts and the fact that functions in the SobolevHilbert space vanish on the boundary, each equation can be rewritten as follows.

$$
\begin{aligned}
\frac{1}{2} \frac{\partial\|u\|^{2}}{\partial \tau}=-D_{1} \int_{W} \nabla u^{2} d \mathbf{x}+r_{1} \int_{W} u^{2} d \mathbf{x}- & \int_{W} \frac{u^{3}}{K} d \mathbf{x}+\int_{W} u \int_{W} A\left(x-x^{\prime}\right) u\left(x^{\prime}, t\right) d \mathbf{x}^{\prime} d \mathbf{x} \\
& +\int_{W} \gamma \operatorname{sgn}(u-v) u^{2} v d \mathbf{x} \\
\frac{1}{2} \frac{\partial\|v\|^{2}}{\partial \tau}=-D_{2} \int_{W} \nabla v^{2} d \mathbf{x}+r_{2} \int_{W} v^{2} d \mathbf{x}- & \int_{W} \frac{v^{3}}{K} d \mathbf{x}+\int_{W} v \int_{W} A\left(x-x^{\prime}\right) v\left(x^{\prime}, t\right) d \mathbf{x}^{\prime} d \mathbf{x} \\
& -\int_{W} \gamma \operatorname{sgn}(u-v) v^{2} u d \mathbf{x}
\end{aligned}
$$

As is clear from the definition of the energy function, equations (21) and (22) yield equation (23) for the derivative of the energy with respect to $\tau$, denoted $\dot{E}$.

$$
\begin{gathered}
\dot{E}=-D_{1} \int_{W} \nabla u^{2} d \mathbf{x}+r_{1} \int_{W} u^{2} d \mathbf{x}-\int_{W} \frac{u^{3}}{K} d \mathbf{x}+\int_{W} u \int_{W} A\left(x-x^{\prime}\right) u\left(x^{\prime}, t\right) d \mathbf{x}^{\prime} d \mathbf{x}+ \\
\int_{W} \gamma \operatorname{sgn}(u-v) u^{2} v d \mathbf{x}-D_{2} \int_{W} \nabla v^{2} d \mathbf{x}+r_{2} \int_{W} v^{2} d \mathbf{x}-\int_{W} \frac{v^{3}}{K} d \mathbf{x}+ \\
\int_{W} v \int_{W} A\left(x-x^{\prime}\right) v\left(x^{\prime}, t\right) d \mathbf{x}^{\prime} d \mathbf{x}-\int_{W} \gamma \operatorname{sgn}(u-v) v^{2} u d \mathbf{x}
\end{gathered}
$$


We will split the terms on the right-hand side of equation (18) into three groups: $\mathrm{P}, \mathrm{I}$, and $\mathrm{N}$. The terms in $\mathrm{P}$ are positive-definite and quadratic, the terms in I will be quadratic, and the terms in $\mathrm{N}$ are the higher-order terms. With these definitions in order, we obtain expressions for $\mathrm{P}$ and $\mathrm{I}$.

$$
\begin{gathered}
P=\|\nabla u\|^{2}+\|\nabla v\|^{2} \\
I=r_{1}\|u\|^{2}+r_{2}\|v\|^{2} \\
N=-\int_{W} \frac{u^{3}}{K} d \mathbf{x}+\int_{W} u \int_{W} A\left(x-x^{\prime}\right) u\left(x^{\prime}, t\right) d \mathbf{x}^{\prime} d \mathbf{x}+\int_{W} \gamma \operatorname{sgn}(u-v) u^{2} v d \mathbf{x} \\
-\int_{W} \frac{v^{3}}{K} d \mathbf{x}+\int_{W} v \int_{W} A\left(x-x^{\prime}\right) v\left(x^{\prime}, t\right) d \mathbf{x}^{\prime} d \mathbf{x}-\int_{W} \gamma \operatorname{sgn}(u-v) v^{2} u d \mathbf{x}
\end{gathered}
$$

Here, we have made use of the definition of the norm and inner product of the space to simplify the relations given. As a result of these definitions, may be rewritten in the form below.

$$
\dot{E}=-P+I+N
$$

We now make another definition to simplify equation (22). Clearly, we have that $\frac{I}{P} \leq \max _{\phi \in H_{0}^{2}(W)} \frac{I}{P}$. To this end, we define a constant $\omega$.

$$
\frac{1}{\omega}=\max _{\phi \in H_{0}^{2}(W)} \frac{I}{P}
$$

With this definition in hand, we obtain equation (29).

$$
\dot{E} \leq-P\left(1-\frac{1}{\omega}\right)+N
$$

We now proceed to bound the terms that comprise N. We begin with $-\int_{W} \frac{u^{3}}{K} d \mathbf{x}$ and the analogous term for $\mathrm{v}$.

$\left|\int_{W} \frac{(u(\mathbf{x}))^{3}}{K} d \mathbf{x}\right| \leq \frac{1}{K}\left(\int_{W}(u(\mathbf{x}))^{4} d \mathbf{x}\right)^{1 / 2}\left(\int_{W}(u(\mathbf{x}))^{2} d \mathbf{x}\right)^{1 / 2} \leq \frac{c_{1}}{K}\|\nabla u\|^{2}\|u\|$

The constant $c_{1}$ arises from the Sobolev inequality. The term involving $v^{3}$ has an identical bound, with a constant $c_{2}$. Next, we consider the terms 
with mixed powers of $\mathrm{u}$ and $\mathrm{v}$.

$$
\begin{gathered}
\left|\int_{W} \gamma \operatorname{sgn}(u(\mathbf{x})-v(\mathbf{x}))(u(\mathbf{x}))^{2} v(\mathbf{x}) d \mathbf{x}\right| \leq \gamma\left|\int_{W}(u(\mathbf{x}))^{2} v(\mathbf{x}) d \mathbf{x}\right| \\
\quad \leq\left(\int_{W}(u(\mathbf{x}))^{4} d \mathbf{x}\right)^{1 / 2}\left(\int_{W}(v(\mathbf{x}))^{2} d \mathbf{x}\right)^{1 / 2} \leq \gamma c_{3}\|\nabla u\|^{2}\|v\|
\end{gathered}
$$

Once again, the analogous term has an identical bound with the role of $\mathrm{u}$ and $\mathrm{v}$ switched, and a new constant $c_{4}$. Finally, we turn to the terms involving the propagator.

$$
\begin{aligned}
& \left|\int_{W} u(\mathbf{x}) \int_{W} A\left(\mathbf{x}-\mathbf{x}^{\prime}\right) u\left(\mathbf{x}^{\prime}, t\right) d \mathbf{x}^{\prime} d \mathbf{x}\right| \\
& \leq\left(\int_{W}(u(\mathbf{x}))^{2} d \mathbf{x}\right)^{1 / 2}\left(\int_{W}\left(\int_{W} A\left(\mathbf{x}-\mathbf{x}^{\prime}\right) u\left(\mathbf{x}^{\prime}\right) d \mathbf{x}^{\prime}\right)^{2} d \mathbf{x}\right)^{1 / 2} \\
& \left.\leq\left(\iint_{W^{2}}\left(A\left(\mathbf{x}-\mathbf{x}^{\prime}\right)\right)^{2} d \mathbf{x}^{\prime} d \mathbf{x}\right)\right)^{1 / 2}\left(\int_{W}(u(\mathbf{x}))^{2} d \mathbf{x}\right)^{1 / 2}\left(\int_{W}\left(u\left(\mathbf{x}^{\prime}\right)\right)^{2} d \mathbf{x}^{\prime}\right)^{1 / 2}
\end{aligned}
$$

We note that the double integral on the right-hand side indicates that the domain $\mathrm{W}$ is being integrated over twice, once for each vector variable. We rename the term involving our kernel $A\left(\mathbf{x}-\mathbf{x}^{\prime}\right)$ to $c_{A}$, since it is a constant that depends only on the choice of kernel for the model.

We have almost bounded $\dot{E}$, we merely note the following relations listed below.

$$
\begin{aligned}
& \|u\| \leq \sqrt{2} E^{1 / 2},\|v\| \leq \sqrt{2} E^{1 / 2} \\
& \|\nabla u\|^{1 / 2} \leq \frac{P}{D_{1}},\|\nabla v\|^{1 / 2} \leq \frac{P}{D_{2}}
\end{aligned}
$$

Using these results, it is simple to show that the derivative obeys the bound given below.

$$
N \leq m P E^{1 / 2}+n E
$$

Here $m=\frac{\sqrt{2} c_{1}}{K D_{1}}+\frac{\sqrt{2} c_{2}}{K D_{2}}+\sqrt{2} c_{3} \gamma+\sqrt{2} c_{4} \gamma$ and $n=4 c_{A}$. Making use of these results, we rewrite equation (35) in the form given below.

$$
\dot{E} \leq-P\left(1-\frac{1}{\omega}-m E^{1 / 2}\right)+n E
$$


In order to obtain our final result, we must make use of one last result regarding functions in the Sobolev-Hilbert space. These functions are known to obey the Poincaré inequality.

$$
\|\nabla \psi\|^{2} \geq \lambda_{1}\|\psi\|^{2}
$$

Here, the $\lambda_{1}$ is understood to be the smallest eigenvalue of the Dirichlet problem $\nabla^{2} \psi=-\lambda \psi$ with $u$ being zero on the boundary of the region. We note that the eigenfunctions of this problem span the space in question, and as such the solutions to the model can be written as a linear combination of them, which will be useful in the remaining analysis to follow. We apply the Poincaré inequality to both $u$ and $v$.

$$
\begin{aligned}
& \|\nabla u\|^{2} \geq \lambda_{1}\|u\|^{2} \\
& \|\nabla v\|^{2} \geq \lambda_{1}\|v\|^{2}
\end{aligned}
$$

We can add these two results to obtain the following equation.

$$
P=D_{1}\|\nabla u\|^{2}+D_{2}\|\nabla v\|^{2} \geq \kappa E
$$

Here, $\kappa=\min \left(2 D_{1} \lambda_{1}, 2 D_{2} \lambda_{1}\right)$. Thus, applying this result to equation (31), we obtain a new bound on .

$$
\begin{aligned}
\dot{E} \leq-P\left(1-\frac{1}{\omega}-m E^{1 / 2}\right)+n E \leq-P & \left(1-\frac{1}{\omega}-m E^{1 / 2}\right)+\frac{n P}{\kappa} \\
& =-P\left(1-\frac{1}{\omega}-m E^{1 / 2}-\frac{n}{\kappa}\right)
\end{aligned}
$$

Thus, we have obtained a result bounding the rescaled time derivative of our energy function in terms of $-P$. Using the Poincaré inequality once more, we can obtain a result in terms of $E$ on the right-hand side. However, we first prove that the term within the parentheses is a positive quantity for all times $\tau$. At the minimum, we must require that $1-\frac{1}{\omega}-\frac{n}{\kappa}>0$.

In order to derive further guarantees, we must first make another assumption regarding the energy function. Following [28], we demand that $0<1-\frac{1}{\omega}-m E^{1 / 2}(0)-\frac{n}{\kappa}$. If we can then prove that $E^{1 / 2}(0)>E^{1 / 2}(t)$ under these circumstances, then the term in parentheses is always positive, and we will have achieved our desired result.

To this end, we apply equation (36) at $\tau=0$. Given our initial assumptions regarding the energy function at this time, we have that at $\tau=0$ $\dot{E}<0$. Now, suppose that we assume that at some time $\tau_{0}$, the term in parentheses on the right-hand side of the inequality is positive. Then, by 
the Intermediate Value Theorem there must be some $\tau_{1}<\tau_{0}$ such that the term in parentheses is zero. For all $\tau$ such that $0<\tau<\tau_{1}$, we must then have that the term in parentheses is negative, and thus on the interval $\left(0, \tau_{1}\right) \mathrm{E}$ is a decreasing function. Therefore, on this interval we have that $E(\tau)<E(0)<\frac{1}{m}\left(1-\frac{1}{\omega}-\frac{n}{\kappa}\right)$. Via another appeal to continuity, we should also have that $E\left(\tau_{1}\right)<E(0)<\frac{1}{m}\left(1-\frac{1}{\omega}-\frac{n}{\kappa}\right)$. However, by assumption $E\left(\tau_{1}\right)$ is equal to the term on the right-hand side of this inequality. So, we have obtained a contradiction and can thus conclude that the term in the parentheses on the right-hand side of equation (36) is always positive, and we can thus simplify the claim to that of equation (42).

$$
\dot{E} \leq-\rho P
$$

Here, we have shown that $\rho$ is always positive by the above argument. Finally, applying the Poincaré inequalty once more, we obtain equation (43).

$$
\dot{E} \leq-\rho \kappa E
$$

So, we have found that given the assumptions detailed above, the constant steady-state solution where both languages die out is a stable steady state. To complete the analysis, we identify when $1-\frac{1}{\omega}-\frac{n}{\kappa}>0$. To this end, we recall the definition of $\omega$. Thus, we maximize the functional $\frac{I}{D}$. The standard method for optimizing a functional is to use the Euler-Lagrange equations [18]. To that end, we apply them to the given functional to obtain two results.

$$
\begin{aligned}
& \omega r_{1} u+D_{1} \nabla^{2} u=0 \\
& \omega r_{2} v+D_{2} \nabla^{2} v=0
\end{aligned}
$$

As was mentioned earlier in the analysis, the eigenfunctions of the Dirichlet problem $\nabla^{2} \psi=-\lambda \psi$ form a basis for functions in the Sobolev-Hilbert space, which we take advantage of here. We expand both $u$ and $v$ in terms of these solutions.

$$
u=\sum_{n=1}^{\infty} u_{n} \psi_{n}, v=\sum_{n=1}^{\infty} v_{n} \psi_{n}
$$

We then substitute these results into the Euler-Lagrange equations and utilize the relationship between the eigenfunctions, eigenvectors, and the operator $\nabla^{2}$. 


$$
\begin{aligned}
& \omega r_{1} \sum_{n=1}^{\infty} u_{n} \psi_{n}=D_{1} \sum_{n=1}^{\infty}{ }_{n} u_{n} \psi_{n} \\
& \omega r_{2} \sum_{n=1}^{\infty} v_{n} \psi_{n}=D_{2} \sum_{n=1}^{\infty}{ }_{n} v_{n} \psi_{n}
\end{aligned}
$$

Since the eigenvalue $\lambda_{1}$ is the smallest, we obtain the following bounds from equations (47) and (48).

$$
\begin{aligned}
& \omega r_{1} \sum_{n=1}^{\infty} u_{n} \psi_{n}>\lambda_{1} D_{1} \sum_{n=1}^{\infty} u_{n} \psi_{n} \\
& \omega r_{2} \sum_{n=1}^{\infty} v_{n} \psi_{n}>\lambda_{1} D_{2} \sum_{n=1}^{\infty} v_{n} \psi_{n}
\end{aligned}
$$

Given these inequalities, we can guarantee our required condition will hold whenever the following inequalities are satisfied.

$$
\lambda_{1}>\frac{\kappa r_{1}}{D_{1}(\kappa-n)}, \lambda_{1}>\frac{\kappa r_{2}}{D_{2}(\kappa-n)}
$$

